\title{
FAIRE FAMILLE AUTREMENT : CE QUE LES MARGES NOUS ENSEIGNENT SUR « LA » PARENTALITE
}

\author{
Virginie Rozée (virginie.rozee@ined.fr)
}

\section{RÉSUMÉ}

Depuis plusieurs décennies dans les sociétés occidentales, on assiste à une transformation de famille et de la parentalité, liée aux changements sociodémographiques et culturels mais aussi aux développement des techniques de reproduction assistée. Ces techniques permettent de concevoir un enfant quelles que soient les caractéristiques, les choix et parcours de vie des futurs parents. Ces parentalités issues des techniques médicales restent néanmoins marginales: elles sont statistiquement minoritaires et ne correspondent pas à la parentalité normative, celle qui est socialement construite et valorisée. L'objectif est de montrer que ces «marges reproductives » sont révélatrices des normes sociales dominantes de la famille et de la parentalité en France ; et de montrer qu'elles représentent un champ d'études en sciences sociales important pour analyser la reproduction de ces normes mais aussi leur transformation.

MOTS CLÉS : marges ; normes sociales ; parentalité

\section{FAZER FAMÍLIA DIFERENTEMENTE : O QUE AS MARGENS NOS ENSINAM SOBRE «A $»$ PARENTALIDADE}

\section{RESUMO}

Depois de muitas décadas, assistimos à transformação da família e da parentalidade nas sociedades ocidentais, ligadas às mudanças sociodemográficas e culturais, mas também ao desenvolvimento de técnicas de reprodução assistida. Essas técnicas permitem conceber uma criança sejam lá quais forem as características, as escolhas e percursos de vida dos futuros pais. Essas parentalidades resultantes de técnicas médicas, no entanto, permanecem marginais: elas são estaticamente minoritáriAs e não correspondem à parentalidade normativa, como àquela que é socialmente construída e valorizada. $\mathrm{O}$ objetivo é de mostrar que essas "margens reprodutivas" são reveladoras das normas sociais dominantes tanto de família quanto de parentalidade na França; e mostrar que elas representam um campo de estudos importante nas ciências sociais para analisar a reprodução dessas normais, mas também suas transformações.

PALAVRAS-CHAVES : margens ; normas sociais ; parentalidade.

\section{INTRODUCTION}

Depuis plusieurs décennies, on observe dans les sociétés occidentales, comme en France, une transformation de la famille et de la parentalité (Golombok 2015 ; Gourarier et Mathieu 2016). Cette évolution s'inscrit dans le cadre des changements démographiques et culturels plus généraux, désignés sous le terme de «deuxième transition démographique » (Van De Kaa 
1987) qui se traduit par un report du mariage et de la parentalité, une augmentation de la cohabitation hors mariage, du divorce et des personnes restant sans enfant (Beaujouan et al. 2017).

Cette transformation de la famille et de la parentalité est également liée aux avancées de la médecine reproductive, en particulier celles qui aident les individus et couples à concevoir un enfant (Courduriès et Herbrand 2014 ; Pulman 2010), désignées ici sous le terme de «techniques de reproduction assistée $»^{1}$. En effet, ces techniques permettent aujourd'hui aux femmes et aux hommes, qui n'envisagent pas une vie sans enfant, de devenir parents et de concevoir quels que soient leurs problèmes de santé, leur infertilité, leurs parcours ou choix de vie. Elles offrent de nouvelles possibilités d'accès à la parentalité, en plus de l'adoption et du confiage (Bonnet 2014).

Pour autant, les normes sociales dominantes du faire famille restent en vigueur en Europe. Ces normes définissent qui peut devenir parents, avec qui et comment : être en couple hétérosexuel stable, ni trop jeune, ni trop âgé et concevoir un enfant au sein de ce couple (Rozée 2015). Il s'agit ici du modèle démographique dominant mais également de celui qui est socialement valorisé (Debest et Mazuy 2014), voir exclusivement accepté dans certains pays. Des hommes, des femmes et des couples décident de faire famille autrement, sans réunir les conditions et les caractéristiques sociodémographiques normatives de la parentalité. Ces individus deviennent parents sans être en couple ou en étant en couple avec une personne de même sexe, sans participer génétiquement ou corporellement à la conception de l'enfant (lorsqu'il y a recours à un donneur de sperme, une donneuse d'ovocytes ou une gestatrice), en ayant plus de 40 ans, etc.

L'objectif est ici de montrer ce que ces marges, à travers le cas de techniques de reproduction assistée, nous enseignent sur les normes sociales dominantes de la famille et de la parentalité en France; et de montrer qu'elles représentent un champ d'études en sciences

\footnotetext{
${ }^{1}$ Les techniques de reproduction assistée regroupent deux principales techniques médicales. (1) L’insémination artificielle : le sperme du conjoint ou d'un donneur (on parle alors d'insémination artificielle avec donneur) est déposé dans l'appareil reproductif de la femme). L'insémination artificielle humaine date du $18^{\text {ème }}$ siècle ([Bateman] Novaes, 1994,), mais elle est aujourd'hui médicalement encadrée et organisée. (2) La fécondation in vitro (FIV) lorsque la fécondation a lieu en éprouvette. Dans ce cas, les embryons, issus des gamètes des parents ou de donneurs/donneuses (donneuse d'ovocytes, donneur de sperme, double don ou accueil d'embryon), sont replacés dans l'utérus de la mère ou d'une autre femme appelée « gestatrice » ou «mère porteuse ». La naissance du premier «bébé éprouvette », appelée Louise Brown, a eu lieu au Royaume-Uni en 1978 (Franklin, 2016). Toutes les autres techniques existantes sont des variantes ou dérivées de la FIV.
} 
sociales important pour analyser à la fois la pérennité des normes sociales dominantes et leur transformation.

\section{LES MARGES EN SCIENCES SOCIALES, REVELATRICES D'UN ORDRE SOCIAL ETABLI ET DE SES CHANGEMENTS}

Les travaux en sciences sociales sur les marges et les marginalités, principalement ceux de l'Ecole de Chicago (Goffman 1968 ; Becker 1985 ; Coulon 2012), portaient sur des groupes qui étaient ou agissaient différemment des codes, règles et normes établies, qui étaient étiquetés comme tels, c'est-à-dire comme « déviants », par les autres groupes «normaux » (par rapport à la norme) ou «dominants ». Le postulat de départ de ces travaux était que les sociétés produisaient leurs propres marges, leurs propres exclusions à travers des logiques de domination, qui définissaient ce qui était socialement approprié ou « normal », et donc, par effet de miroir, ce qui ne l'était pas. D'autres lectures des marges sont possibles comme le montre Michel Wieviorka, sociologue, directeur d'études à l'Ecole des hautes études en sciences sociales (EHESS) de Paris, lors de la Conférence inaugural du colloque « la reconnaissance des marges, au cœur des dynamiques sociales en France et au Japon » à Paris en 2016.

Une première approche est de considérer les marges, comme des victimes d'un ordre social ou de dominations, et comme pouvant jouer un rôle dans le changement social, dans la transformation des normes ou des règles. Les marges annoncent ici l'apparition d'un nouvel ordre établi, d'un nouvel acteur social. Michel Wieviorka évoque l'exemple du mouvement ouvrier à la fin du $19^{\text {ème }}$ siècle : la révolution industrielle fait apparaître de nouvelles « classes laborieuses », de nouveaux acteurs collectifs (les ouvriers), considérés comme « marginaux » par rapport à la bourgeoisie de l'époque et au prolétariat. Pourtant, l'apparition de ces « marginaux » annonce le développement de la société industrielle et la naissance de la classe ouvrière qui sera bientôt la classe sociale majoritaire.

Une deuxième approche est d'appréhender les marges comme traduisant « une exigence morale que le reste de la population n'a pas le courage, la possibilité, ou la volonté d'exprimer » (Wieviorka 2016). Les marges sont ici d'ordre moral (exemple des dissidents de l'Union Soviétique dans les années 60-70).

Enfin, les marges peuvent être empreintes de créativité, en résistant et transformant l'ordre et les normes établies. Ici, les marges sont avant-gardistes et culturelles : les artistes maudits par exemple ou plus généralement les artistes ou intellectuel·le·s qui sont a priori 
considérés comme marginaux, mais dont la créativité, en rupture avec les œuvres classiques du moment, peut représenter, a posteriori, un nouveau courant artistique ou de pensée (exemple de l'impressionnisme ou du romantisme).

Ces différentes lectures sociologiques des marges peuvent s'appliquer à une même réalité sociale. Les marges sont un révélateur de ce qui est socialement considéré comme approprié et majoritairement accepté (les normes sociales dominantes) mais aussi des changements sociaux en cours dans une société donnée.

Dans l'introduction de leur ouvrage collectif La France des marges (2017), Martine Candelier-Cabon et Solène Gaudin résume parfaitement l'intérêt d'étudier les marges et leur relation à la norme.

\begin{abstract}
S'intéresser aux marges, n'est-ce pas vouloir scruter l'inconnu? (...) ce qui est questionné ici, c'est la construction et le rapport à la norme, comme un «canon » porté et développé par le centre, nécessairement et/ou indirectement dominant (le «mainstream» dans la littérature américaine), celui qui s'impose par le fait. (...) Ces marges qu'elles soient spatiales et/ou sociales ne sont [donc] pas figées, elles peuvent constituer et même incarner des situations de transition, de sas, d'interstice spatial comme temporel. Ainsi, ces espaces souvent présentés comme repliés, en dehors du système, sont également porteurs de formes alternatives, innovantes et créatives, sous l'effet de dynamiques plurielles, endogènes comme exogènes (Candelier-Cabon et Gaudin 2017 : 7-9).
\end{abstract}

Les marges révèlent en effet les pérennités des normes : les actions et conduites sont, dans une société donnée, déterminées par les normes dominantes et les représentations collectives majoritaires. Mais, on observe partout et toujours des arrangements, des stratégies de contournement et de résilience qui font bouger les normes. Ainsi, les actions et conduites, y compris celles observées depuis les marges, sont l'effet d'intériorisation des normes que l'on contribue à construire mais aussi à déconstruire. Madeleine Gauthier (1994) écrit à ce sujet que les marges remettent en question l'ordre ancien et contribuent à reconstruire un nouvel ordre. «Qu'elle soit excentrique ou qu'elle soit excluante, [la marginalité] fait partie du processus même du changement dans les sociétés » (Gauthier 1994 : 178).

Cette approche par les marges fait écho aux divers travaux de Michel Foucault, résumés dans l'article de Bruno Lautier (2006). L'objectif de Michel Foucault, écrit-il,

était un objectif de méthode : montrer qu'on comprend une société non pas en faisant une analyse de l'intérieur, mais depuis les marges : les fous, les malades, les criminels, les pervers, ne nous apprennent pas grand-chose sur eux-mêmes, mais beaucoup sur nous (Lautier 2006 : 19). 


\section{Nanduty}

ISSN:2317-8590

\section{LES MARGES GENREES DANS LE CHAMP DE LA SEXUALITE ET DE LA REPRODUCTION}

En sciences sociales, et notamment dans les études de genre, des travaux se sont intéressés aux marges pour mieux appréhender le système de genre dans sa dimension intersectionnelle, prenant en compte la diversité des rapports sociaux de domination (sexe, classe, race) ${ }^{2}$.

Dans le champ de la sexualité, l'approche par les marges peuvent permettre de mieux appréhender les normes dominantes de genre. En France, Mathieu Trachman s'est intéressé au travail pornographique, un travail marginal, analysé comme un révélateur des rapports sociaux de sexe et de l'hétéronormativité (Trachman 2013). Michel Bozon a, quant à lui, exploré les normes de genre et de la sexualité au prisme de l'âge, et notamment à la sexualité chez les plus de 50 ans, soit une population peu étudiée en matière de pratiques sexuelles car socialement considérée comme peu active. Pourtant, les représentations et pratiques sexuelles de la population du $3^{\text {ème }}$ âge montrent à la fois une reproduction des normes sexuelles dominantes mais aussi d'importants changements dans le temps, qui sont liés à l'évolution des conditions et de la place des femmes dans la société française (Bozon et Rennes 2015). Virginie Ringa a, de son côté, mené une étude la sexualité des femmes ménopausées en France. Elle a montré que, contrairement à ce qui est généralement affirmé, les changements hormonaux de la ménopause n'affectent pas négativement la vie sexuelle des femmes. L'altération de vie sexuelle avec l'âge des femmes résulte en réalité principalement des représentations négatives de genre liées à la vieillesse (Ringa et al. 2013).

Dans les études de genre, les recherches sur la reproduction à partir des marges se sont multipliées ces dernières décennies avec, notamment, le développement des techniques de reproduction assistée, comme la FIV, le don de gamète ou la gestation pour autrui. Ces techniques permettent de pallier une infertilité médicalement constatée (lorsqu'un couple hétérosexuel souhaitant devenir parent, ayant des relations sexuelles non protégées, ne parvient pas à concevoir un enfant) mais également ce qui est communément appelée une «infertilité sociale » (lorsque, la situation sociale et familiale ne permet pas la conception d'un enfant), les deux types d'infertilité ne s'excluant pas mutuellement. Ces techniques permettent donc de nouvelles façons de concevoir, de devenir parents et donc de faire famille.

\footnotetext{
${ }^{2}$ Voir par exemple l'ouvrage de hooks 2017.
} 


\section{Nanduty}

ISSN:2317-8590

Leur utilisation reste néanmoins marginale en nombre. Aux Etats-Unis, en 2013, les naissances d'enfants issus d'une technique de reproduction assistée représentaient $2 \%$ du nombre total des naissances (Centers for Disease Control and Prevention et al. 2015). En France, en 2014, 3,1\% des enfants ont été conçus avec une technique de reproduction assistée (Agence de la Biomédecine 2015). Si cette proportion n'est pas négligeable et bien qu'elle soit en constante augmentation depuis plusieurs décennies (La Rochebrochard 2018), elle reste fortement minoritaire par rapport aux conceptions par relations sexuelles.

Faire famille en utilisant des techniques de reproduction assistée est donc une réalité marginale, puisque cela concerne des hommes, des femmes et des couples dont les projets procréatifs et parcours parentaux sont différents de ceux majoritairement observés et statistiquement minoritaires. Ce faire famille marginal est néanmoins révélateur des normes dominantes de la parentalité, en particulier l'hétéronormativité, de leur intériorisation mais également de leur transformation. Pour reprendre les mots de Rayna Rapp, «lorsque la reproduction devient problématique [lorsqu'elle est, comme ici, hors-norme, marginale], elle fournit une lentille à travers laquelle les normes culturelles, les luttes et les transformations peuvent être vues $»^{3}(\operatorname{Rapp} 2001: 466)$.

\section{LES MARGES REPRODUCTIVES : LE CAS DES COUPLES DE FEMMES ET DES FEMMES SEULES}

Les couples de femmes et les femmes seules (qui ne sont pas en couple) recourant à des techniques de reproduction assistée pour devenir mères, sont particulièrement révélatrices des pérennités et transformations de ces normes en France. Elles constituent ici ce que nous appelons des « marges reproductives », qui sont par définition des marges genrées puisqu'elles ne réunissent pas les prérogatives normatives de genre du faire famille (à savoir ici concevoir un enfant dans une configuration conjugale hétérosexuelle). Mais elles sont également en marge du système légal puisqu'en France, jusqu'à aujourd'hui, la loi de bioéthique, qui encadre l'accès et les modalités de recours aux techniques de reproduction assistée, ne permet pas à ces femmes de recourir à ces techniques médicales pour devenir parents. En effet, ces techniques sont uniquement destinées aux couples hétérosexuels ayant une infertilité médicale (en théorie, puisque de nombreuses infertilités sont en fait idiopathiques). Les couples de même sexe et les

\footnotetext{
${ }^{3}$ Traduction par l'auteure de: "When reproduction becomes problematic, it provides a lens through which cultural norms, struggles, and transformations can be viewed" (Rapp 2001: 466).
} 
femmes seules se rendent donc à l'étranger, principalement en Belgique ou en Espagne, pour bénéficier d'un don de sperme, d'un double don ou d'un accueil d'embryon (Rozée et La Rochebrochard 2013). Depuis 2018, néanmoins, le nouveau projet de loi de bioéthique envisage de permettre à toutes les femmes qui le souhaitent de recourir en France à une aide médicale à concevoir (Mathieu 2020).

Les couples de femmes, comme les couples d'hommes, qui réalisent un projet parental en France, contreviennent à la norme hétérosexuelle de la parentalité. Cette norme correspond à la majorité des parentalités en France. En 2009, France Prioux et Didier Breton estimait que 91\% des enfants âgés de 0 à 3 ans vivaient avec leurs deux parents de sexes différents (Breton et Prioux 2009). Dans les années 1970, «sortir du placard» ou «faire son coming out » signifiait renoncer à la parentalité. Mais à partir des années 1990, les couples de même sexe revendiquent le droit à être parents par adoption ou en utilisant les techniques de reproduction assistée.

Virginie Descoutures a montré comment les familles homoparentales de femmes qu'elle a interrogées ont intériorisés certains éléments des normes dominantes de la parentalité, ce qu'elle définit comme les «résidus de conformité à la norme hétérocentrée » (Descoutures 2010 : 95) comme l'intraconjugalité et la filiation biologique.

Il y a en premier lieu une construction conjugale du désir d'enfant et la recherche d'un modèle familial « classique » et « triangulaire », à deux parents et un (voire plusieurs) enfant(s). La recherche de cette intraconjugalité familiale s'observe lorsque les femmes recourent à un don de sperme puisque nombreuses sont celles qui optent pour un don anonyme, inhibant ainsi la présence du tiers dans la conception de l'enfant.

Dans le même temps, ce choix fait écho à une autre norme dominante dans la société française, celle qui sacralise le lien génétique et la filiation biologique, puisque les mères lesbiennes définissent souvent le géniteur (le donneur de sperme) comme le père de l'enfant. Effet des normes sociales dominantes intériorisées, cet amalgame entre donneur et père (que l'on retrouve également chez les femmes seules) fait que l'anonymat peut apparaître comme une tranquillité de l'esprit, comme une façon de préserver la stabilité de la famille, sans avoir à redouter une revendication paternelle future du donneur.

Les femmes ont intériorisé ces représentations normatives mais, dans leur pratique, elles les transgressent puisqu'elles optent pour une famille certes à deux parents mais deux parents de même sexe, et font famille en faisant primer la filiation sociale et non biologique. Virginie Descoutures écrit : 


\section{Nanduty}

s'intéresser aux familles homoparentales peut être tout à fait éclairant pour continuer de penser les 'transformations' de la société : le cadre dans lequel l'institution familiale s'inscrit, ses normes et ses transformations accompagnent, en même temps qu'elles en sont l'indicateur, les changements plus larges de la société (Descoutures 2010 : 147).

De la même façon, les femmes qui ne sont pas en couple et qui décident de concevoir un enfant en recourant à une aide médicale contreviennent à l'hétéronormativité de la parentalité. Cette réalité de la «monomaternité choisie » issue des techniques de reproduction assistée est nouvelle et peu étudiée. Elle a été récemment mise en lumière dans le cadre des débats sur la révision de la loi de bioéthique. Cette réalité est statistiquement minoritaire dans l'ensemble des familles françaises mais également parmi les familles monoparentales davantage issues d'une rupture conjugale (Toulemon 2005 ; Eydoux et Letablier 2007).

Loin d'un projet féministe de vouloir construire sciemment une famille sans père, la décision de recourir à un donneur est souvent justifiée par l'absence d'un père potentiel et comme une option plus honnête et plus saine que d'autres alternatives. Le témoignage d'Elsa, publié dans $L$ 'OBS le 4 octobre 2017 sous le titre «PMA : 'A 41 ans, j'ai fait un enfant toute seule. C'était tout sauf un caprice' », incarne parfaitement les résultats des deux études menées en France sur ces maternités solo (Rozée 2013 : Mehl 2016). Il s'agissait pour les femmes interrogées, qui ne concevaient pas une vie sans enfant, de devenir mère avant qu'il ne soit trop tard (c'est-à-dire lorsqu'elles ne seront plus fertiles ou socialement considérées comme « trop âgées » pour être mère), dans l'attente d'une mise en couple avec l'homme qui deviendra alors le père social de l'enfant. La situation de monomaternité est donc espérée provisoire.

Les histoires et discours de ces femmes soulignent une forte intériorisation du modèle normatif de la parentalité, celui qui se décline ici en fonction de l'âge et de la situation conjugale (Rozée, 2015), modèle auquel elles s'apprêtent pourtant à contrevenir puisqu'elles décident d'avoir un enfant seules, en ayant recours à un don de gamètes.

\section{CONCLUSION}

Etudier les discours et expériences d'individus qui empruntent des chemins différents de ceux majoritairement empruntés par la population pour devenir parents permet d'analyser les normes dominantes de la parentalité et de la famille, comment elles se reproduisent mais aussi comment elles évoluent, se déconstruisent et se reconstruisent. Le faire famille marginal révèle 


\section{Nanduty}

ISSN:2317-8590

l'expression, souvent intériorisée, de ces normes mais également les moyens mis en œuvre pour à la fois s'y conformer et s'y soustraire.

Ces résiliences et résistances accompagnent les changements normatifs liés à l'émergence de nouvelles parentalités dans les sociétés occidentales telles que l'homoparentalité, la monoparentalité, la parentalité sociale, la parentalité tardive, ou encore la pluriparentalité. Les marges en question, de plus en plus visibles dans la société française, montrent surtout que les parentalités peuvent être et sont aujourd'hui, en France comme ailleurs, complexes et diverses et que le faire famille ne se conjugue désormais plus au singulier mais revêt des réalités sociales plurielles.

\section{RÉFÉRENCES}

AGENCE DE LA BIOMÉDECINE. 2015. Activité d'Assistance Médicale à la Procréation 2014. In : Agence de la biomédecine - Le rapport annuel médical et scientifique 2015 (agencebiomedecine.fr). (Acessado em 14set21).

[BATEMAN] NOVAES, Simone. 1994. Les passeurs de gamètes, Nancy, Presses Universitaires de Nancy.

BEAUJOUAN, Eva ; SOBOTKA, Tomáš ; BRZOZOWSKA, Zuzanna ; ZEMAN Krystof. 2017. "Has childlessness peaked in Europe?", Population \& Societies, 540, p.1-4

BECKER, Howard. 1985. Outsiders. Etudes de sociologie de la déviance, Paris, a.m. Métailié (collection «Observations »)

BONNET, Doris. 2014. «Adopter un enfant dans le contexte de la Procréation médicalement assistée en Afrique subsaharienne », Cahiers d'Etudes Africaines, LIV(3), 215, p.769-786

BOZON, Michel ; RENNES, Juliette. 2015. «Histoire des normes sexuelles : l'emprise de l'âge et du genre », Clio. Femmes, Genre, Histoire, 42

BRETON, Didier ; PRIOUX, France. 2009. « Observer la situation et l'histoire familiale des enfants », in REGNIER-LOILIER, Arnaud (dir.). Portraits de familles. L'enquête Étude des relations familiales et intergénérationnelles, Paris, Ined, p.143-167

CANDELIER-CABON, Martine; GAUDIN, Solène (dir.). 2017. La France des marges, Rennes, Presses universitaires de Rennes

CENTERS FOR DISEASE CONTROL AND PREVENTION; AMERICAN SOCIETY FOR REPRODUCTIVE MEDICINE; SOCIETY FOR ASSISTED REPRODUCTIVE TECHNOLOGY. 2015. Assisted Reproductive Technology National Summary Report 2013. Atlanta (GA), US Dept of Health and Human Services

COULON, Alain. 2012. L'Ecole de Chicago, Paris, Presses universitaires de France (collection «Que sais-je »)

COURDURIES, Jérôme; HERBRAND, Cathy. 2014. "Genre, parenté et techniques de reproduction assistée : bilan et perspectives après 30 ans de recherche », Enfances, Familles, Générations, 21, p.i-xxvii

DEBEST, Charlote; MAZUY Magali. 2014. "Childlessness: a life choice that goes against the norm”, Population and Societies, 508, p.1-4. 
DESCOUTURES, Virginie. 2010. Les Mères lesbiennes, Paris, PUF/Le Monde (collection « Partage du savoir »)

EYDOUX, Anne ; LETABLIER Marie-Thérèse. 2007. Les familles monoparentales en France, Rapport de recherche, Centre d'études de l'emploi, 36

FRANKLIN, Sarah. 2016. "Louise Brown: my life as the world's first test-tube baby", Reproductive Biomedicine \& Society Online, 3, p.142-144

GAUTHIER, Madeleine. 1994. «Entre l'excentricité et l'exclusion: les marges comme révélateur de la société », Sociologie et sociétés, 262, p.177-188

GOFFMAN, Erving. 1968. Asiles. Etude sur la condition sociale des malades mentaux et autres reclus, Paris, Les Editions de Minuit (collection « Le sens commun »)

GOLOMBOK, Susan. 2015. Modern Families. Parents and Children in New Family Forms, Cambridge university press

GOURARIER, Mélanie; MATHIEU, Séverine. 2016. «Des parentés contemporaines?», Journal des anthropologues, 144-145, p.13-27

HOOKS, bell. 2017 [1984]. De la marge au centre. Théories féministes, Paris, Edition Cambourakis (collection Sorcières)

LA ROCHEBROCHARD (de), Elise. 2018. « 1 enfant sur 30 conçu par assistance médicale à la procréation en France », Population\&Société, 556

LAUTIER, Bruno. 2006. « Discussion (suite) notes d'un sociologue sur l'usage de la notion de 'marge' dans les sciences sociales du développement », Revue Tiers Monde, 185, p.17-20

MATHIEU, Séverine. 2020. Faire famille aujourd'hui. PMA, bioéthique et religion, Paris, Le Cavalier bleu

MEHL, Dominique. 2016. Maternités solo, Editions universitaires européennes

PULMAN, Bertrand. 2010. Mille et une façons de faire les enfants. La révolution des méthodes de procreation, Paris, Calmann-Lévy

RAPP, Rayna. 2001. "Gender, Body, Biomedicine: How Some Feminist Concerns Dragged Reproduction to the Center of Social Theory", Medical Anthropology Quartely, 15(4), p.466477

RINGA, Virginie; DITER, Kevin; LABORDE, Caroline; BAJOS, Nathalie. 2013. "Women's Sexuality: From Aging to Social Representations", The journal of sexual medicine, 10(10), p.2399-2408

ROZEE, Virginie. 2013. «Elles font des bébés toutes seules », Terrain, 61, p.134-149

ROZEE, Virginie. 2015. "Les normes de la maternité en France à l'épreuve du recours transnational de l'assistance médicale à la procréation », Recherches Familiales, 12, p.43-55

ROZEE, Virginie. 2018. Les paradoxes genrés de la reproduction transnationale, Mémoire d'HDR, Paris, Université Paris 1 - Panthéon Sorbonne

ROZEE, Virginie ; LA ROCHEBROCHARD (de), Elise. 2013. "Cross-border reproductive care among French patients: experiences in Greece, Spain and Belgium", Human Reproduction, 28(11), p.3103-3110

TOULEMON, Laurent. 2005. "Who are the late mothers?", Revue d'Épidémiologie et de Santé Publique, 53(HS2), p. 2S13-24

VAN DE KAA, Dirk J. . 1987. "Europe's second demographic transition”, Population Bulletin, 42(1), p.1-57

WIEVIORKA, Michel. 2016. «Ce que sont les marges aux sciences sociales », Hypothèses, disponible sur https://wieviorka.hypotheses.org/706 